\title{
Investigation of Equity in Arrival Time of Emergent Accidental Patient to Hospital in (2018-2019) in a City in North of Iran
}

Mohaddese Omidi

Guilan University of Medical Sciences

Behzad Zohrevandi

Guilan University of Medical Sciences

Enayatollah Homaie Rad ( $\sim$ homaierad@gmail.com )

Guilan University of Medical Sciences

Research article

Keywords: Equity, EMS, Gini index

Posted Date: August 17th, 2020

DOI: https://doi.org/10.21203/rs.3.rs-54240/v1

License: (c) (i) This work is licensed under a Creative Commons Attribution 4.0 International License.

Read Full License 


\section{Title page:}

\section{Investigation of Equity in Arrival Time of Emergent Accidental Patient to Hospital in (2018- 2019) in a City in North of Iran}

\section{Mohaddese Omidi}

Student Research Committee, Guilan Road Trauma Research Center, Guilan University of Medical Sciences, Rasht, Iran

\section{Behzad Zohrevandi}

Guilan Road Trauma Research Center, Guilan University of Medical Sciences, Rasht, Iran

\section{Enayatollah Homaie Rad}

Corresponding Author, Social Determinants of Health Research Center, Guilan University of Medical Sciences, Rasht, Iran

Email: homaierad@gmail.com

Tel: +981333332498

Cell: +989120558446 


\title{
Investigation of Equity in Arrival Time of Emergent Accidental Patient to Hospital in (2018- 2019) in a City in North of Iran
}

\begin{abstract}
Background: Fairness is an important and controversial issue when allocating public resources. People need to be arrived early to the hospitals when they are injured in traffic accidents. Both the mean and equity of the time of arriving to the hospital are important. The aim of this study was to investigate of equity in arrival time of emergent accidental patient to hospital in 2018-2019 in a city in North of Iran.

Methods: This study was a Descriptive-analytical study. In this study we extracted the information from Guilan province trauma system data for Poursina Hospital in Rasht in 2018 and 2019. Data included information on age, sex, level of education, place of residence, time of transfer from the scene of the accident to the hospital, type of vehicle, marriage and occupation. The Gini coefficient was used to calculate equity in access to pre-hospital emergency services and regression models were estimated for inequity in time to receive hospital services.

Results: The study showed that the mean and standard deviation of patients' arrival time from the time of the accident to the time of arrival at the hospital was $64.48 \pm 47.63$ minutes (minimum 9 minutes and maximum 462 minutes). Gini coefficient was 0.31 ( $\mathrm{p}<0.001$ ) which shows in the Rasht city has a relative and appropriate equity when the patient arrives. The contribution of each factor using the regression model indicated the existence of a part of the inequity due to the way the patient was transferred. The transfer time of patients by car was 40 minutes longer (p-value <0.001) and by other methods (except ambulance and car) was 26 minutes longer $(\mathrm{p}=0.036)$. In children, the transfer time was 42 minutes less $(p=0.003)$. Other variables did not explain the inequity $(p>0.05)$

Conclusion: According to the time of arrival of patients and Gini index, in Rasht, equity in providing services is in a good condition. This indicates that the emergency department does not systematically transport people to the hospital late.
\end{abstract}

Keywords: Equity ، EMS ، Gini index 


\section{Background}

One of the most important aspects of health care is pre-hospital care, which is responsible for care before the patient arrives at the hospital $(1,2)$. In Iran, up to 30 million missions are performed annually in 4500 pre-hospital emergency bases, of which about $15 \%$ of these missions are related to trauma patients and in traffic accidents. In Iran, the death toll from accidents is higher than the total death toll from the three diseases of AIDS, tuberculosis and malaria. 9\% of the total mortality in Iran and 12\% of the disease burden is due to accidents, especially traffic accidents(3). Traffic accidents are the main cause of loss of life and after strokes and heart attacks, are the third leading cause of death in Iran. The goal of the prehospital emergency is to provide effective and accessible emergency care to all those who need it, which can be done by distributing justice and a fair distribution of resources between different groups in the community(3). Studies have shown that distributive justice indices and health indices are related to each other and with the improvement of distributive justice indices, health indices also increase(4). Justice in the field of health means that if one needs health services, can access them at the right time and enough $(5,6)$. The availability of health care resources and medical equipment is one of the most important issues in the health system that the World Health Organization and the Organization for Economic Cooperation and Development have considered it necessary to measure justice in its distribution $(5,7)$. Just as health and medical care are important and necessary in health, rooting out the social factors involved in endangering health also improves indicators that are far from the global standard (8). And as much as economic growth and its fair distribution are important in the health of society, one of the important strategies for economic growth is investing in health and related factors. This means that by improving the health situation and supporting the deprived people, we will also improve the economic level of the society (9). EMS requires continuous quality assessment of its performance indicators to provide the best out-of-hospital care. In 2001, the National Academy of Medicine (NAM), formerly known as the Medical Institute (IOM), identified six quality areas for system improvement. Health care products identified: safety, time-oriented, effectiveness, patient-centered, efficiency and justice (10). The operation of the EMS system depends on a variety of decisions, including the design of the service area, the allocation of appropriate ambulance locations, and dispatch policies. Maximum response time and coverage are the criteria used to measure the efficiency of the EMS system (11). To be arrived to the hospital injured people can use their own car, Emergency Medical Services (EMS) and etc. Emergency medical systems (EMS) are public services that often provide the first line of response to the immediate needs of health care in the community (12). Of course, justice is an important and controversial issue when allocating public resources (11). As mentioned before, an important factor in determining the performance of EMS along with the quality of medical care provided is the timely delivery of services or the prompt response time by EMS, which can mean the difference between life and death (12). With the passage of time and the progress made in various fields, EMS also faced challenges along with the opportunities (13). Emergency medical services are also the first level of response to timesensitive situations. Therefore, it has special importance. By better managing EMS needs and planning, we will have a better understanding of consumer needs and can reduce the differences in the benefits of different subgroups (14). As mentioned, one of the most important ways for economic growth is to invest 
in the field of health and related factors, and this means that by improving the health situation and supporting the deprived, we will also improve the economic level of society (9).

The main purpose of this study is investigating of equity in arrival time of accidental emergent patients at the hospital in 2018 and 2019 in Rasht, Iran. The present study helps policy makers and managers of the health system to have a clear view of the state of justice when emergency patients arrive at a hospital in Rasht. Since the average indicators are general indicators, having justice indicators can clearly explain the differences at the time of arrival of the pre-hospital emergency.

\section{Methods}

\section{Subject and data collection}

This study is a descriptive-analytical and retrospective study. The study population is all accidental patients in Rasht who referred to the hospital between March 2018 and March 2019 (The Persian year of 1398).

In this study, all registered cases were used. Inclusion criteria for being added in the data bank contained: The sample should have been referred to the hospital due to a car accident, information about it should have been entered in the trauma system of Guilan province, important and main variables related to it should have been available, and the accident should have taken place in Rasht.

Individuals with incomplete information in the files were excluded from the study. At the end, 1067 data were available. In this study, information about the variables of age, sex, level of education, place of residence, time of transfer from the place of accident to the hospital, type of vehicle, marriage and occupation were collected from the trauma system databank of Guilan province for Poursina Hospital in Rasht.

\section{Evaluation}

In this study, the Gini coefficient is used to calculate equality in access to hospital services form the accident place. And regression models were used to calcualte the contribution of each factor in creating injustice in access to emergency services.

The Gini coefficient is used to measure inequalities in social and economic studies and measures the distribution of income and health distributions across different classes of society. The Gini coefficient is a value between zero and one, which indicates the value of zero, the minimum and the value of one, the maximum of dispersion and inequality in the distribution of health indicators. If this coefficient goes to zero, the society is closer to equity, and if it goes to 1 , it is closer to inequity. The Gini coefficient formula is as follows:

$$
\text { Gini }=\frac{\sum_{i=1}^{n} \sum_{j=1}^{n}\left|r_{i}-r_{j}\right|^{\alpha}}{2 n^{2} \mu^{\beta}}
$$


$r_{i}=$ The health status of the individual or group $\mathrm{i}$

$r_{j}=$ The health status of the individual or group $\mathrm{j}$

$\mu=$ Average health status for the whole community

$\mathrm{n}=$ Number of people in the study

$\alpha=2$ and $\beta=2$

The Lorenz curve is a cumulative frequency curve. This curve shows the inequality index on the coordinate axis ( $\mathrm{Y}$ and $\mathrm{X}$ ) along with a line with an angle of 45 degrees (fair line). If the distribution of health in terms of social components in society is perfectly fair, the Lorenz curve sticks to the fair line, and if the distribution of health in society is completely unjust, the Lorenz curve becomes much more curved so that it coincides with the X-axis. As a result, as much as the Lorenz curve is closer to fair line, there is less inequality and the society is closer to justice. Then we enter each of the variables into the regression model to determine the contribution of each of them in creating inequality in percentage.

\section{Result:}

The obtained data showed that the mean and standard deviation of patients' arrival time from the time of accident to the time of arrival at the hospital was $64.48 \pm 47.63$ minutes, with a minimum arrival time of 9 minutes and a maximum time of 462 minutes. (table1)

Gini coefficient of patient arrival time from accident to hospital in the study sample was equal to 0.31 ( $\mathrm{p}$ $<0.001$ ) which shows that the calculated Gini coefficient is statistically significant (table 2).

The Gini coefficient of the type of transport that patients used to get to the hospital is equal to 0.27 for those patients who used an ambulance; It was 0.44 for those who came to the hospital by car and 0.37 for others (table3).

Figure 1 shows the findings of the study in relation to the Lorenz curve for arrival time. In this graph, a line called the fair line and a curve called the Lorenz curve are observed. The distance between them shows the degree of injustice in the study sample. Therefore, according to the fig1, it can be concluded that the arrival time of accidental patients in Rasht is relatively fair.

Figure 2 shows Lorenz curve of type of vehicle that patient used to get to the hospital. The comparison between the curves shows that those patients who used the ambulance to get to the hospital were closer to equity and those who were transported by car were farther away from equity. 
Table 6 shows the status of time distribution and related factors by using the regression model. As shown in the table, part of the injustice occurred due to the way the patient was transferred, which means that if the patient was transferred by car, the patient transfer time is 40 minutes longer and the coefficient of the mentioned variable is statistically significant. (P-value $<0.001$ ) and if the patients are transported by other methods (except ambulance and car), the transfer time is 26 minutes longer. Here, too, the coefficient of the relevant variable is statistically significant $(\mathrm{p}=0.036)$. (table6)

In children, the transfer time of patients is less than 42 minutes, which indicates that the time for children to reach the emergency room of hospital is shorter. Here, too, the coefficient of the relevant variable is statistically significant $(\mathrm{P}=0.003)$.

Since p-value in other variables is above 0.05 , these variables do not explain the injustice when reaching the emergency.

\section{Discussion}

In this study, the Gini coefficient of the patient's transfer time from the time of the accident to the time of arrival at the hospital was equal to 0.31 ( $\mathrm{p}<0.001)$, which P-value shows that the calculated Gini coefficient is statistically significant. The Gini coefficient is a value between zero and one, which indicates the value of zero, the minimum and the value of one, the maximum of dispersion and inequality in the distribution of health indicators. In other words, how this coefficient is closer to zero, the society is closer to justice, and how much it is closer to the 1 it is farther from justice. Therefore, considering that the calculated Gini coefficient was equal to 0.31, it can be concluded that the transfer time of patients from the time of the accident to the time of arrival at the hospital in Rasht is relatively fair. Yalan Liu et al. conducted a study in 2015 in Chongqing, China, to evaluate equity in the need, use and distribution of EMS and related resources. In this study the concentration index, was used to assess equality in the distribution of needs and the use of EMS among three regions with different GDPs, and the Gini index was used to assess equity in the demographic and geographical distribution of EMS facilities and human resources. The findings of this study showed that patients in developed areas were more likely to use EMS than those living in less developed areas (CI: 0.296 to 0.423). On the other hand, the geographical distribution of EMS facilities and human resources in Chongqing City (Gini 0.4 to 0.5 ) indicates relative justice but a large gap in different areas. Therefore, in this city, justice in the needs, operation, distribution and allocation of EMS resources is relatively low and the provision of such services has not met all the needs of patients (15).

Also in a study by Mr. Wei Chen and et al.in 2015 in Gong Dong, China, to assess justice in the operation and distribution of pre-hospital emergency care resources, the Gini index in 
ambulances, first aid stations and dispatch centers Based on the geographical area was 0.4402, $0.4696,0.4214$, respectively, which indicates the relative justice in using these facilities in different areas in Gong Dong city(16).

In a similar study, K Yan and et al. In 2017 in China investigate the equity in EMS emergency medical services, using and distributing resources equity. They use the concentration Index (CI) to measure equality and equity in the need for EMS and the extent of its use and utilization in the western, central and eastern regions of China and the Gini coefficient of population distribution and geographical distribution of facilities were used to assess the degree of justice in the allocation of resources. The results showed that trauma-related mortality (CI: range from 0.1241 to 0.1504) was consistently concentrated in poor areas, and this inequality became more pronounced among different areas year after year. Distribution and allocation of emergency medical services in geographical distribution (Gini from 0.5481 to 0.5696 ) also indicates the existence of injustice as well as the existence of a large gap between regions (13).

In the present study, out of 1063 injured subjects, 202 (19\%) were female and $861(81 \%)$ were male. The mean and standard deviation of the arrival time of patients from the time of accident to arrival at the hospital, for the gender of the sample, was $61.05 \pm 3.25$ in females and $65.29 \pm 1.64$ in males. By using the regression model to determine the contribution of this variable in creating injustice, considering that the gender of people with a p-value of 0.277 , it can be concluded that this variable did not explain the injustice when arriving in the emergency room in Rasht. In a study conducted by Mr. Park et al. in South Korea in 2020 with the aim of examining justice in the access of the elderly (65 years and older) to EMS, and in which they examined various variables, 391 of the 3175 people studied (12.31\%) were male and 2784 (87.69\%) were female, that $11.65 \%$ of men and $54.14 \%$ of women used an ambulance. In this study, the results showed that predictive factors such as age played an important role in making a difference in the use of EMS in the elderly in South Korea(14).

In a study conducted by Mr. Sang Do-shin et al. In 2012 in South Korea to examine the relationship between patients' gender and the results of outpatient cardiac arrest, a total of 13,922 people (over 20 years of age with a Underlying heart problem), 8764 (62.9\%) were male and $5158(37.1 \%)$ were female. The results showed that women had shorter response time (7.5 minutes vs. 8 minutes) and shorter transfer time (23.7 minutes vs. 24.6 minutes) than men (P $<0.001)$ but overall they had less than men basic life support $(70.8 \%$ vs. $77.5 \%)$ or use an external automatic defibrillator $(9.6 \%$ vs. $14.3 \%)$ or advanced cardiac life support (42.2\% vs. 49.2\%) (17) .

In the present study, out of 1063 samples, 419 of them were single (39.42\%) and 644 of them were married (60.58\%). The mean and standard deviation of the transfer time of patients from the time of accident to arrival at the hospital for marital status is $65.98 \pm 2.40$ minutes for single people and $63.52 \pm 1.84$ minutes for married people. In determining the contribution of this variable in creating injustice using the regression model and considering that the marital status of 
people with a p-value of 0.745 , it can be concluded that this variable also has not been explained the injustice in arrival time in Rasht.

In a study conducted by Mr. Park et al. in South Korea in 2020 with the aim of examining justice in the access of the elderly (65 years and older) to EMS, out of a total of 3175 people, 1742 $(54.87 \%)$ were married and 1433 (\%45.13) made up other people (single, divorced and widowed). $29.92 \%$ of married people and $35.87 \%$ of other people used emergency services. Which has shown its effective role in the use of EMS in the elderly in South Korea(14).

In the present study, out of a total of 1063 samples, 195 people lived in rural areas (18.34\%) and 868 people $(81.66 \%)$ lived in cities. The mean and standard deviation of the transfer time of random patients from the time of accident to arrival at the hospital per residence is $68.17 \pm 2.77$ minutes in rural residents and $63.67 \pm 1.68$ minutes in Rasht residents, whose p-value after Being in the regression model was equal to 0.297 , which indicates that this variable did not explain the injustice in arrival time in Rasht. However, in a study conducted by Mr. Park and his colleagues, out of a total of 3175 samples, 1107 (34.87\%) were residents of the capital and 2068 (65.13\%) were residents of areas other than the capital. Among them, 24.19\% of people living in the capital and $41.61 \%$ of other people have used EMS services, which after being included in the multivariate logistic regression analysis model, it was found that the residence of people also plays an important role in the use of EMS in the elderly in Korea(14).

In the present study, the mean and standard deviation of the age of the injured was $39.28 \pm 18.7$ years (minimum age 2 years and maximum age 89 years).Examining the contribution of this variable in creating injustice showed that in Rasht, the age of individuals had no effect on the time of transfer of patients from the time of the accident to the time of arrival at the hospital $(\mathrm{p}=$ $0.097)$

In a study conducted by Mr. Park et al. In South Korea in 2020 with the aim of examining justice in the access of the elderly (65 years and older) to EMS, the results showed that variables such as health status and the presence of chronic disease and variables Items such as gender, marital status and level of education have played a decisive role in the use of EMS by the elderly(14).

\section{Conclusions}

Findings of the study showed that due to the time of arrival of patients and Gini index, in Rasht city, justice in providing services was in a good condition. On the other hand, the results of the study showed that although the distribution is fair in this study, the average transfer time of patients from the time of the accident to the time of arrival at the hospital is long. 


\section{Abbreviations}

EMS: Emergency Medical Services

AIDS: Acquired Immune Deficiency Syndrome

NAM: National Academy of Medicine

IOM: Medical Institute

GDP: Gross Domestic Product

CI: concentration Index

\section{Declaration}

The authors confirm that the data have been gathered and analyzed at Guilan Road Trauma Research Center, Guilan University of Medical Sciences, Rasht, Iran.

\section{Ethics approval and consent to participate}

The study was confirmed ethiclay by deputy of research, Guilan University of Medical Sciences and consent of participants was confirmed during data gathering.

\section{Consent for Publication}

The authors have the consent for publication of the results.

\section{Availability of data and material}

Data is available for all of readers who wants to use the data.

\section{Competing interests}

The authors declare that they have no conflicts of interest.

\section{Funding}

None.

\section{Authors' contributions}


EHR gathered data, analyzed data and supervised the article, MO wrote the article, reviewed the literarture and BZ critically reviewed the article.

\section{Acknowledgements}

This study was derived from the Medical Doctorate Thesis of Mrs Mohaddese Omidi. Authors thanks to Guilan Road Trauma Research Center for its supports.

\section{References}

1. Aboueljinane $L$, Sahin $E$, Jemai Z. A review on simulation models applied to emergency medical service operations. Computers \& Industrial Engineering. 2013;66(4):734-50.

2. Heydari H, Kamran A, Zali ME, Novinmehr N, Safari M. Customers' satisfaction about prehospital emergency medical services in Lorestan, Iran. Electronic Physician. 2017;9(3):3974.

3. hadian $\mathrm{m}$, abedinejad $\mathrm{m}$, Seyedin $\mathrm{H}$, behrooj s. Relationship between Justice and Performance Indicators of Iran Pre-Hospital Emergency. Journal of Modern Medical Information Sciences. 2020;5:3140.

4. Babakhani M, Ghasemi R, Rafiey H, Raghfar H, Biglariyan A. The Relationship Between Distributive Justice and Health Indicators in Iran. Social Welfare Quarterly. 2012;12(46):259-78.

5. Hurst J, Hughes M. Performance Measurement and Performance Management in OECD Health Systems. Disponível em www1. oecd org OECD Health Systems Labour Market and Social PolicyOccasional Papers. 2001;47(2001):1-60.

6. Organization WH. The world health report 2000: health systems: improving performance: World Health Organization; 2000.

7. WHO G. The world health report 2002: reducing risks, promoting healthy life. Education for Health (Abingdon, England). 2002;16(2):230.

8. Malek Afzali Hossein NS, Ayazi Seyed Mohammad Hadi. Indicators of Justice in Health 2016.

9. Translators: Dr. Fayyaz Bakhsh Ahmad DOMA, Dr. Poyandjoo Morvarid, Dr. Forouzan Setareh.

Removing injustice in a generation: Policy Council of the Ministry of Health and Medical Education; 1390. $264 \mathrm{p}$.

10. Moafa H, Kuijk Sv, Franssen G, Moukhyer M, Haak H. What is known about the quality of out-ofhospital emergency medical services in the Arabian Gulf States? A systematic review. PloS one. 2019;14(12):e0226230.

11. Enayati S, Mayorga ME, Toro-Díaz H, Albert LA. Identifying trade-offs in equity and efficiency for simultaneously optimizing location and multipriority dispatch of ambulances. International Transactions in Operational Research. 2019;26(2):415-38.

12. Chanta S, Mayorga ME, McLay LA. Improving emergency service in rural areas: a bi-objective covering location model for EMS systems. Annals of Operations Research. 2014;221(1):133-59.

13. Yan K, Jiang Y, Qiu J, Zhong X, Wang Y, Deng J, et al. The equity of China's emergency medical services from 2010-2014. International journal for equity in health. 2017;16(1):10.

14. PARK J, Sohn A. Equity of Access to Emergency Medical Services Utilization by Elderly Koreans. 2020.

15. Liu Y, Jiang $Y$, Tang S, Qiu J, Zhong X, Wang Y. Analysis of the equity of emergency medical services: a cross-sectional survey in Chongqing city. Int J Equity Health. 2015;14:150.

16. Chen W, Huang C, Guo W, Li Z, Xu C, Cao Y. The Equity of Pre-Hospital Emergency Care at the County Level in Guangdong, China: A Cross-Sectional Study, 2015. SM J Public Health Epidemiol. 2017;3(2):1044. 
17. Ahn KO, Do Shin S, Hwang SS. Sex disparity in resuscitation efforts and outcomes in out-ofhospital cardiac arrest. The American journal of emergency medicine. 2012;30(9):1810-6.

\section{Figure Legends}

Figure 1. Lorenz curve of total arrival time. If the red line places closer to the blue line (equity line) it is considered that there is complete equality in time to arrival.

Figure 2. Lorenz curve of arrival time when the patient uses EMS services (red line) his/her own car (green line) and others (yellow car). If these lines places closer to the blue line (equity line) it is considered that there is complete equality in time to arrival. 
Figures

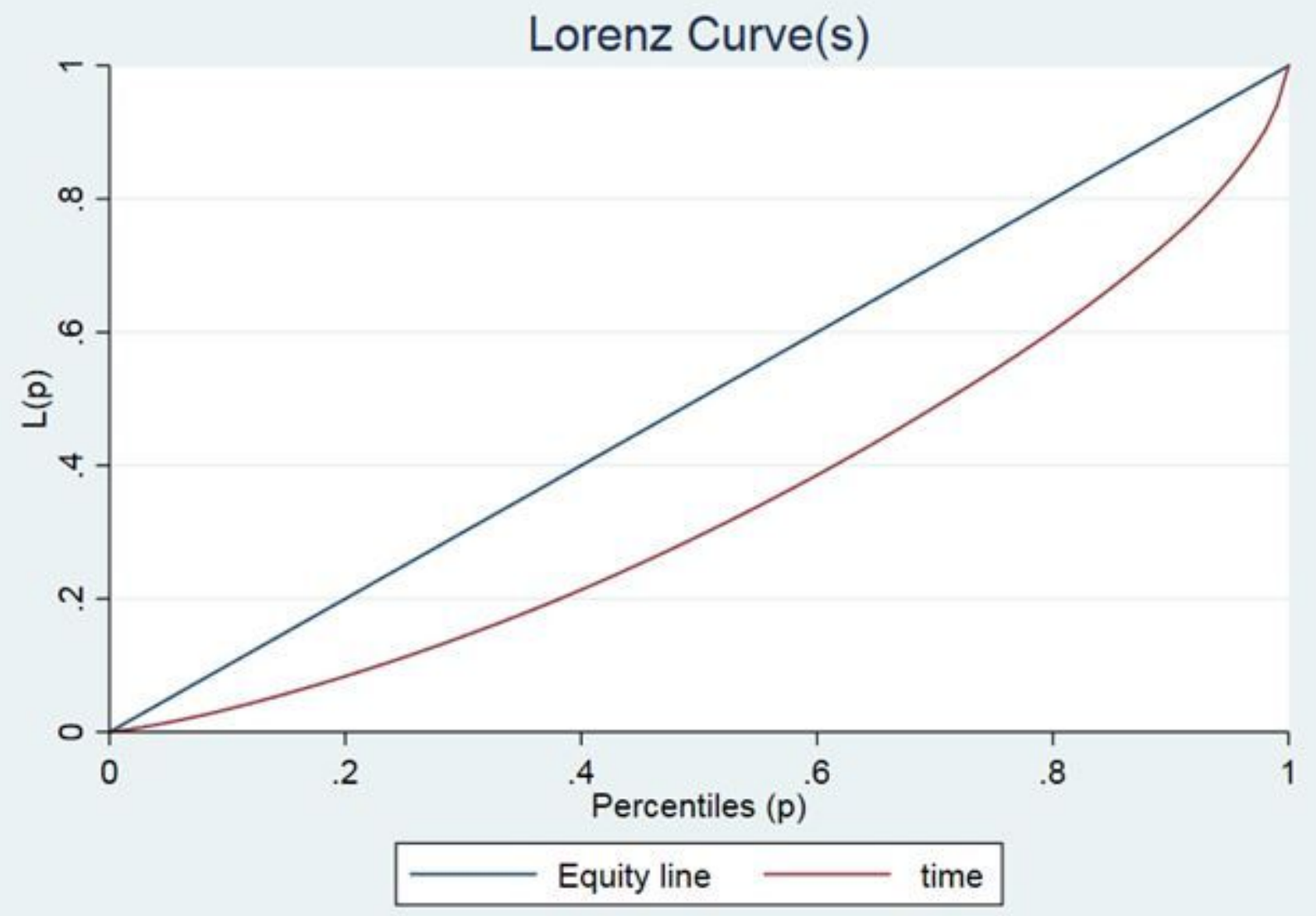

Figure 1

Lorenz curve of arrival time 


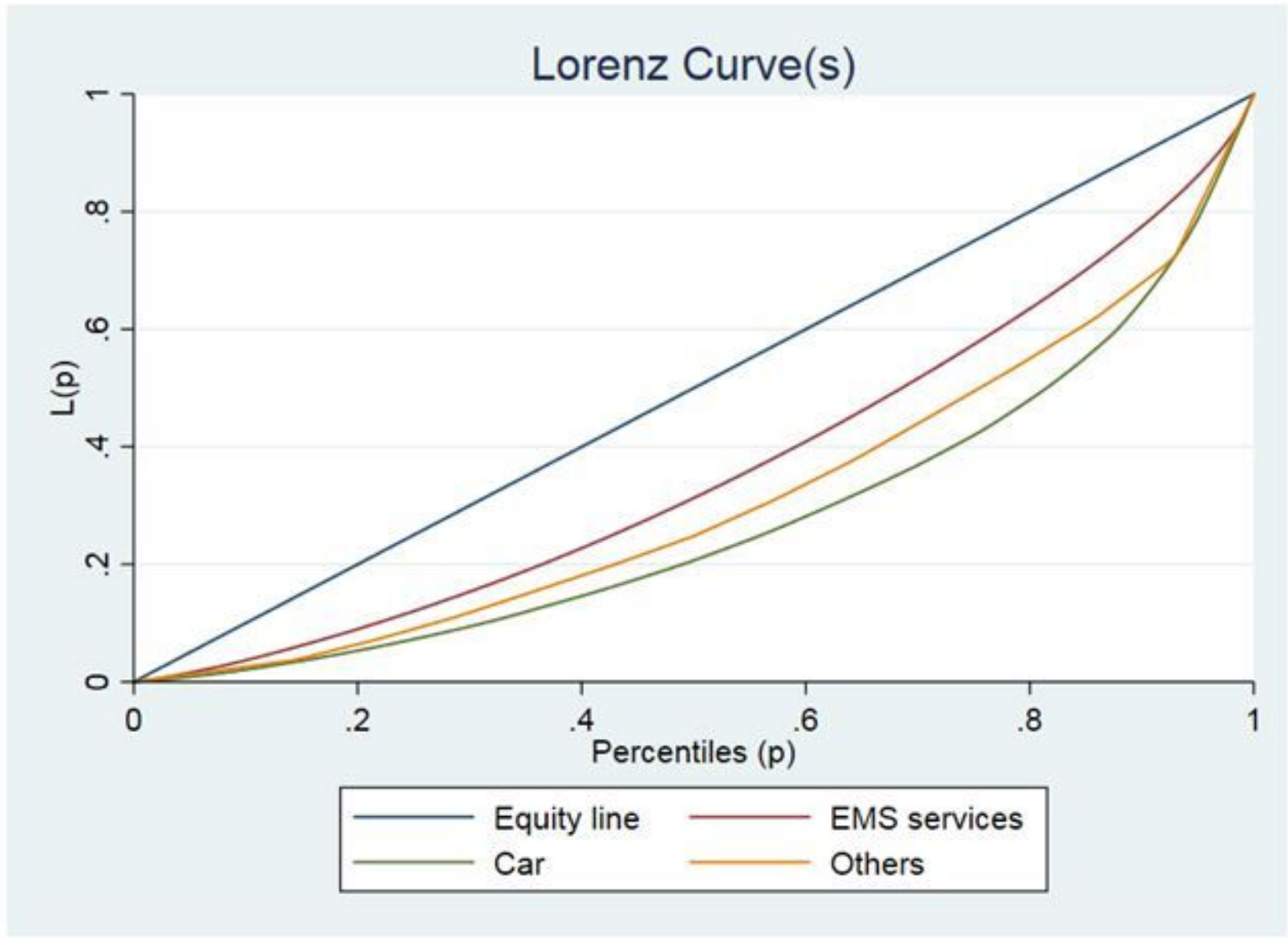

Figure 2

Lorenz curve by type of vehicle. Group1: ambulance. Groupe2: car. Group3: other 